\title{
Effect of secondary lymphoid tissue chemokine suppression on experimental ulcerative colitis in mice
}

\author{
H. Zhang*, X. Zhang*, X. Ding, W. Cao, L. Qu and G. Zhou \\ Department of Gastroenterology, \\ The Affiliated Hospital of Nantong University, Nantong, China \\ *These authors contributed equally to this study. \\ Corresponding author: G. Zhou \\ E-mail: zhouguoxiong@medmail.com.cn
}

Genet. Mol. Res. 13 (2): 3337-3345 (2014)

Received January 25, 2013

Accepted June 18, 2013

Published April 29, 2014

DOI http://dx.doi.org/10.4238/2014.April.29.12

\begin{abstract}
The secondary lymphoid tissue chemokine (CCL21) is closely associated with lymphoid homing and anti-tumor immune responses. CCL21 also has a chemotactic effect on intestinal lymphocytes. This study mainly focused on CCL21 expression in experimental ulcerative colitis and on the effects of CCL21 suppression on this disease in mice. The mouse colitis model was induced by dextran sulfate sodium (DSS) in 40 female BALB/c mice that were equally distributed into five groups: control, DSS, propylene glycol, triptolide (TL), and dexamethasone treatment groups. The disease activity index, general morphology score of the colon, and histological pathology score of colon tissues were evaluated. CCL21 expression was examined in colons of mice by immunohistochemistry, reverse transcription-polymerase chain reaction, and Western blotting analysis. CCL21 was upregulated in the mouse model of ulcerative colitis (control group vs DSS group/ propylene glycol group, $\mathrm{P}<0.01$ ). The TL and dexamethasone treatments improved colitis symptoms and decreased CCL21
\end{abstract}


expression (TL group/dexamethasone group vs DSS group/ propylene glycol group, $\mathrm{P}<0.05)$. In conclusion, CCL21 was shown to be involved in the induction of ulcerative colitis. Suppression of CCL21 expression decreased damage induced from ulcerative colitis, indicating that CCL21 targeted therapy might be an effective treatment for this disease.

Key words: Ulcerative colitis; Secondary lymphoid tissue chemokine; Triptolide; Dexamethasone

\section{INTRODUCTION}

The incidence and prevalence of ulcerative colitis has rapidly increased in China recently, and has now reached a rate of 116 cases per million people. Ulcerative colitis is becoming one of the major causes of common intestinal diseases and chronic diarrhea (Ouyang et al., 2005). The secondary lymphoid tissue chemokine CCL21 is a cysteine-cysteine chemokine (C-C chemokine), which is closely associated with lymphoid homing and anti-tumor immune responses. CCL21 also has chemotactic effects on intestinal lymphocytes. During ulcerative colitis, CCL21 is upregulated in the intestine and induces the migration of CCR7 positive lymphocytes into lymph nodes. Furthermore, it induces homing of several kinds of lymphocytes, and promotes the activation and differentiation of antigen-presenting cells (APC) (Debes et al., 2002; Stein et al., 2003; Weninger et al., 2003; Thanarajasingam et al., 2007). In this study, the effects of CCL21 suppression on ulcerative colitis were examined in mice. Furthermore, we evaluated whether inhibition of CCL21 expression might have a therapeutic effect on ulcerative colitis.

\section{MATERIAL AND METHODS}

\section{Animals and grouping}

A total of 40 female Balb/c mice weighing 22-24 g, and aged between seven to eight weeks, were purchased from the Laboratory Animal Center, Yangzhou University. Animals were randomized into five groups, each consisting of eight animals, including the control, the dextran sulfate sodium (DSS, MP Biomedicals, USA), the propylene glycol, the triptolide (TL, Zelang Medical Science and Technology Co., Ltd. Nanjing, China), and the dexamethasone groups. Following methods of Stevceva et al. (Stevceva et al., 2001, te Velde et al., 2007), all animals were provided free access to 5\% DSS solution (5 g DSS in $100 \mathrm{~mL}$ distilled water) for seven days, except for the control group that had access to distilled water only. From the third to the seventh day, mice in the propylene glycol group were administered with $2 \%$ propylene glycol [ $0.2 \mathrm{~mL}$, intraperitoneal (ip), quantum dot (qd) injection], the triptolide group was administered $0.6 \mathrm{mg} / \mathrm{kg}$ TL in $2 \%$ propylene glycol $(0.2 \mathrm{~mL}, i p, \mathrm{qd})$, and the dexamethasone group was administered $0.4 \mathrm{mg} / \mathrm{kg}$ dexamethasone $(0.2 \mathrm{~mL}$, ip, qd). On the eighth day, the mice were sacrificed by cervical dislocation in order to collect the colon specimens. All animal experiments were approved by the ethical committee for Animal Care and Use of the Affiliated Hospital of Nantong University. 


\section{Symptom assessment and evaluation}

The disease activity index (DAI) was determined by scoring the extent of body weight loss, stool consistency, and gross bleeding, according to the method described by Murano et al. (2000). The formula to calculate DAI was as follows: (combined score of weight loss, stool consistency, and bleeding)/3. The DAI evaluation was performed by assigning number scores based on these three factors, which included $0=$ no weight loss, normal stool consistency, and no bleeding; $1=1-5 \%$ weight loss, loose stool consistency, and Guaiac $(+)$ bleeding; $2=5-10 \%$ weight loss, loose stool consistency, and Guiac (+) bleeding; $3=10-15 \%$ weight loss, loose stool consistency, and Guiac $(+)$ bleeding; $4=$ more than $15 \%$ weight loss, diarrhea, and gross bleeding.

\section{General morphology evaluation}

After cervical dislocation, mice were immediately dissected in order to remove the colon. The intestine was cut along the mesentery, and the stool was eliminated. The intestine was washed with saline solution several times. The general morphology of the colon was observed and recorded according to the following criteria: $0=$ no damage; $1=$ mucosal congestion; $2=$ ulceration area less than $25 \%$ of the damaged area; $3=$ ulceration area equal to $25-50 \%$ of the damaged area; $4=$ ulceration area more than $50 \%$ of the damaged area.

\section{Histological evaluation}

Tissues with severe damage were removed and paraffin-embedded. They were then cut into tissue sections and stained with hematoxylin and eosin (HE). Stained sections were examined blindly in order to find evidence of colitis (Boirivant et al., 2001). The criteria used to assess colitis were as follows: $0=$ no evidence of inflammation; $1=$ very low level of lymphocyte infiltration of less than $10 \%$ in a high-power field (hpf); 2 = low lymphocyte infiltration of $10-25 \%$ in hpf; 3 $=$ moderate level of lymphocyte infiltration $(25-50 \%$ in hpf) with high vascular density and thickening of the bowel wall; $4=$ high level of lymphocyte infiltration (> 50\% in hpf), high vascular density, crypt elongation with distortion, and transmural bowel wall thickening with ulceration.

\section{Immunohistochemistry (IHC)}

IHC was performed using the LAB VISION 2D automatic immunohistochemical staining instrument with an EnVision two-step staining. The rabbit IgG anti CCL21 (Abcam, USA) (1:100) and goat IgG anti rabbit IgG (1:100) antibodies were used for incubation. Sections were observed under the same conditions, and each slice was counted in at least 10 fields under 400X magnification. Cells with brownish-yellow or brown granules in the nucleus or cytoplasm were considered as positive cells. Evaluation was assessed as the percentage of positive cells among all cells observed: $0=$ negative cells; $1=1-25 \%$ positive cells; $2=26$ $50 \%$ positive cells; $3=51-75 \%$ positive cells, and $4=76-100 \%$ positive cells.

\section{Reverse transcription-polymerase chain reaction (RT-PCR)}

The total RNA from each mouse colon was extracted using Trizol (Invitrogen, 
USA). After measuring the concentration, the total RNA with a $260 / 280$ ratio of $1.8-2$ was stored at $-80^{\circ} \mathrm{C}$. Forward and reverse primer sequences used to amplify the CCL21 and $\beta$ actin were synthesized by the Sangon Biotech Co., Ltd., Shanghai. The forward and reverse primers for CCL21 (204 bp) were as follows: 5'-GCAAGAACCAAGTTTAGGCTGT-3' and 5'-CCCTTTCCTTTCTTTCCAGACT-3'. The forward and reverse primers for $\beta$-actin (446 bp) were 5'-GAGACCTTCAACACCCCAGC-3' and 5'-CCACAGGATTCCATACCCAA-3'. The mRNA portion of the total RNA was used as the template. The thermal conditions for reverse transcription were as follows: $37^{\circ} \mathrm{C}$ for $15 \mathrm{~min}$ and $85^{\circ} \mathrm{C}$ for $5 \mathrm{~s}$. The cDNA was used for PCR or was stored at $-20^{\circ} \mathrm{C}$. The thermal cycling conditions for PCR were $10 \mathrm{~min}$ at $95^{\circ} \mathrm{C}$ for pre-denaturation, followed by 45 cycles of denaturation at $95^{\circ} \mathrm{C}$ for $10 \mathrm{~s}$, annealing at $60^{\circ} \mathrm{C}$ for $20 \mathrm{~s}$ ( $\beta$-actin, CCL21), and extension at $72^{\circ} \mathrm{C}$ for $20 \mathrm{~s}$.

\section{Western blotting}

The expression of CCL21 was measured by Western blotting. The total tissue protein was extracted and its concentration was measured by UV spectrophotometry. For sodium dodecyl sulfate-polyacrylamide gel electrophoresis (SDS-PAGE), $20 \mu \mathrm{L}$ protein sample was loaded in each well. Proteins were transferred onto a nitrocellulose membrane and blocked for $4 \mathrm{~h}$ in 5\% Tris-buffered saline with Tween-20 (TBST). The CCL21 antibody was used at a concentration of 1:2500 at $4^{\circ} \mathrm{C}$ overnight and washed in TBST for 10 min five times. Subsequently, the second antibody was used at a concentration of 1:5000 for $2 \mathrm{~h}$, and the bands were visualized by enhanced chemiluminescence (ECL). The data were processed with a gel imaging analysis system and the normalized expression level of CCL21 was measured as the gray scale ratio of CCL21 to $\beta$-actin bands.

\section{Statistical analysis}

Experimental results were entered into an Excel sheet to be used in a database in the future. The results are reported as means \pm standard deviation (SD) in SPSS 17.0. Differences among groups were tested using one-way analysis of variance (ANOVA). A P value lower than 0.05 was considered to be statistically significant.

\section{RESULTS}

\section{DAI evaluation}

Mice in the control group exhibited normal activity, diet, and stool consistency, along with normal hair color and weight gain. However, DAI was increased in mice in the DSS and propylene glycol groups. These mice exhibited anorexia, a reduction in daily activity, drab hair color, and weight loss with a loose stool from day two to three. On the 4th day, these mice had significantly higher DAI compared to the control group with bloody stool and significant weight loss. They barely moved and exhibited an arch-shaped back. TL and dexamethasone treatment (ip) increased the feeding and body weight relative to mice in the DSS and propylene glycol groups. After the treatment, mice exhibited a better hair color, less bloody stool, and less DAI increase (Table 1). 


\begin{tabular}{|c|c|c|c|c|c|}
\hline Day & Control group DAI & DSS group DAI & Propylene glycol group DAI & TL group DAI & DXM group DAI \\
\hline 1 & 1 & 1 & 1 & 1 & 1 \\
\hline 2 & 1 & 1.50 & 1.4 & 1.30 & 1.40 \\
\hline 3 & 1.45 & 2.20 & 2.05 & 1.90 & 2.10 \\
\hline 4 & 1 & 2.65 & 2.50 & 2.60 & 2.60 \\
\hline 5 & 1 & 2.96 & 3.1 & 2.3 & 2.10 \\
\hline 6 & 1 & 3.25 & 3.22 & 2.2 & 1.90 \\
\hline 7 & 1 & 3.68 & 3.53 & 2.1 & 1.80 \\
\hline
\end{tabular}

$\mathrm{DSS}=$ dextran sulfate sodium; $\mathrm{TL}=$ triptolide $\mathrm{DXM}=$ dexamethasone.

\section{General morphology observation and evaluation}

There was no evidence of edema, hyperemia, erosion, or ulceration in the colonic mucous membranes of mice in the control group. The mice in the DSS and propylene glycol groups showed severe erosion and superficial ulceration in their colon, especially in the distal colon. There was moderate mucosal edema, hyperemia, mild edema, and hyperemia in the proximal colon. The TL and dexamethasone treatments alleviated these symptoms, resulting in less erosions and ulcerations in the colons of mice (Table 2).

Table 2. Histological disease scores; morphology scores; Immunohistochemical scores (means $\pm \mathrm{SD}$ ).

\begin{tabular}{lccc}
\hline Group $(\mathrm{N}=8)$ & Histological disease scores & Morphology scores & Immunohistochemical scores \\
\hline Control group & $0.43 \pm 0.10$ & $0.65 \pm 0.15$ & $1.00 \pm 0$ \\
DSS group & $2.82 \pm 0.41^{\mathrm{a}}$ & $3.16 \pm 0.32^{\mathrm{a}}$ & $3.56 \pm 0.28^{\mathrm{a}}$ \\
Propylene glycol group & $2.88 \pm 0.50^{\mathrm{a}}$ & $3.40 \pm 0.20^{\mathrm{a}}$ & $3.38 \pm 0.18^{\mathrm{a}}$ \\
TL group & $1.82 \pm 0.22^{\mathrm{bc}}$ & $2.22 \pm 0.32^{\mathrm{bc}}$ & $2.06 \pm 0.19^{\mathrm{bc}}$ \\
DXM group & $1.65 \pm 0.21^{\mathrm{bc}}$ & $1.98 \pm 0.19^{\mathrm{bc}}$ & $1.98 \pm 0.25^{\mathrm{bc}}$ \\
\hline
\end{tabular}

DSS group and Propylene glycol group compared with control group ${ }^{\text {a }}<0.01$; TL group and DXM group compared with control group ${ }^{\mathrm{b}} \mathrm{P}<0.05$; TL group and DXM group compared with DSS group and Propylene glycol group ${ }^{\mathrm{C}} \mathrm{P}$ $<0.05$. DSS, dextran sulfate sodium; TL, triptolide; DXM, dexamethasone.

\section{Histological observation and evaluation}

HE staining of control mice tissues showed no evidence of damage or erosion in the colonic mucosal epithelium, and the glandular morphology was normal. However, there was a small amount of inflammatory cell infiltration in the submucosa. In contrast, the DSS and propylene glycol groups showed severe damage and ulceration in the colonic mucosal epithelium with inflammatory cell infiltration in the mucosa, and the architecture of the crypts was distorted $(\mathrm{P}<0.01)$. Treatment groups showed reduction in the levels of congestion and edema compared with those of the DSS and propylene glycol groups $(\mathrm{P}<0.05)$, but symptoms were still worse than mice in the control group $(\mathrm{P}<0.05)$ (Figure 1; Table 2).

\section{IHC}

CCL21 expression was examined by IHC staining in the intestinal mucosa of mice in each group. The CCL21 expression level significantly increased in the DSS and propyl- 
ene glycol groups $(\mathrm{P}<0.01)$ compared to that of the control group (Figure 2$)$. The TL and dexamethasone treatments significantly decreased CCL21 expression relative to the DSS and propylene glycol groups $(\mathrm{P}<0.05)$. However, mice in the TL and dexamethasone treatment groups exhibited higher CCL21 expression compared to that of the control group $(\mathrm{P}<0.05)$ (Table 2; Figure 1).

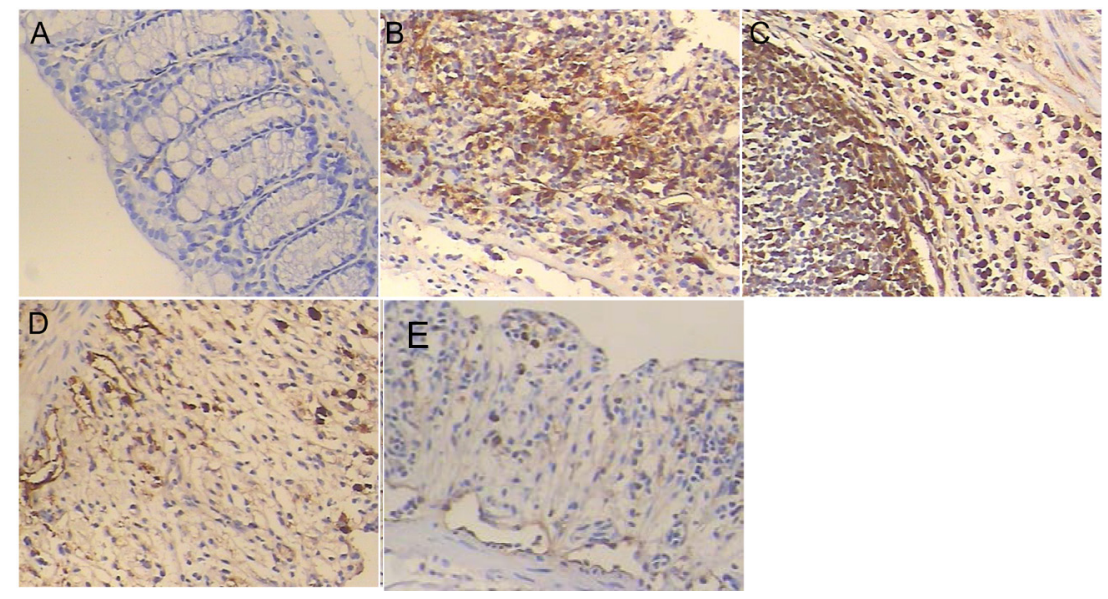

Figure 1. CCL21 protein expression detected by immunohistochemistry in experimental colitis in mice. A. CCL21 protein in control group; B. CCL21 protein in DSS group. DSS, dextran sulfate sodium; C. CCL21 protein in Propylene glycol group; D. CCL21 protein in TL group, TL, triptolide; E. CCL21 protein in DXM group. DXM = dexamethasone.

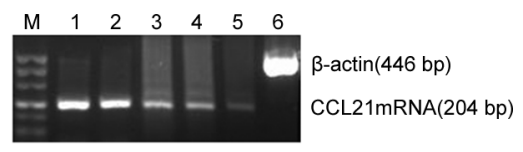

Figure 2. CCL21 mRNA expression detected by RT-PCR. Lane $M=$ Mark; lane $1=$ CCL21 mRNA in DSS group. DSS $=$ Dextran sulfate sodium; lane $2=$ CCL21 mRNA in Propylene glycol group; lane $3=$ CCL21 mRNA in TL group. $\mathrm{TL}=$ Triptolide; lane $4=\mathrm{CCL} 21 \mathrm{mRNA}$ in DXM group. $\mathrm{DXM}=$ Dexamethasone; lane $5=\mathrm{CCL} 21 \mathrm{mRNA}$ in control group; lane $6=\beta$-actin mRNA.

\section{RT-PCR}

The CCL21 gene expression level was significantly increased in the DSS and propylene glycol groups $(\mathrm{P}<0.01)$ compared to that of the control group. The TL and dexamethasone treatment significantly decreased the upregulation of CCL21 expression compared to the DSS and propylene glycol groups $(\mathrm{P}<0.05)$. However, both $\mathrm{TL}$ and dexamethasone treatment groups exhibited higher CCL21 expression compared to that of the control group $(\mathrm{P}<0.05)$ (Figure 2).

\section{Western blot}

The protein expression level of CCL21 was significantly increased in the DSS and 
propylene glycol groups $(\mathrm{P}<0.01)$ compared to the control group. The TL and dexamethasone treatments significantly decreased CCL21 expression relative to those of the DSS and propylene glycol groups $(\mathrm{P}<0.05)$ (Figure 3$)$.

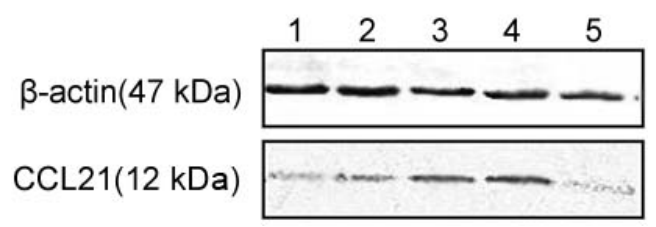

Figure 3. CCL21 protein expression detected by Western blotting. Lane $1=$ CCL21 protein in DXM group. DXM $=$ Dexamethasone; lane $2=$ CCL21 protein in TL group. TL = Triptolide; lane $3=$ CCL21 protein in Propylene glycol group; lane $4=$ CCL21 protein in DSS group. DSS $=$ Dextran sulfate sodium; lane $5=$ CCL21 protein in control group.

\section{DISCUSSION}

CCL21 is a CC chemokine that originates from the endothelium. The CCL21 gene is located on human chromosome 9p13. CCL21 is the first chemokine that was demonstrated to participate in lymphocyte homing (Riedl et al., 2003). Gunn et al. (1999) demonstrated that CCL21 was mainly expressed in the lymph nodes, high endothelium venules (HEVs) in the peyer assembly areas, stromal cells in the T-cell district, and in the peripheral regions of lymphatic vessel endothelial and lymphoid follicles.

CCL21 has chemotactic effects on T, B, NK, and dendritic cells (DC) to peripheral lymphatic organs, and participates in several types of immune responses (Riedl et al., 2003; Stein et al., 2003; Liu et al., 2005; Legler et al., 2006; Ashour et al., 2007; Thanarajasingam et al., 2007; Gollmer et al., 2009; Fedele et al., 2011). Ashour et al. (2007) reported that subcutaneous injection of CCL21 induced assembly of cytotoxic T lymphocytes (CTL) in draining lymph nodes, mainly the $\mathrm{CD}^{+}, \mathrm{CD}^{+}$, and $\mathrm{CD} 62^{-}$subpopulations. The amount of DC and CTL reached a peak by the 4th day following CCL21 injection, indicating that CCL21 assembled the DC and CTL and provided the conditions enabling DC to uptake antigens and improve Tcell immune responses, which is crucial for the activation of anti-inflammatory and anti-tumor immune responses. Wiede et al. (Veerman et al., 2007; Wiede et al., 2007) also reported that tumor necrosis factor-dependent CCL21 over-expression in mice induced dysplasia of nonspecific lymphatic tissues due to alteration of the T-cell assembly.

DCs, which are expressed in the gastrointestinal epithelium, make contact with bacteria that are internalized through the digestive tract. However, the attraction of DC by CCL21, which is expressed in the lymphoid tissue of the gastrointestinal tract, results in the spread of the bacteria in DCs (Cheminay et al., 2002; Watanabe et al., 2007; Zimmerman et al., 2008; Britschgi et al., 2010; Murphy, 2010; Haessler et al., 2011; Tal et al., 2011). Kawashima et al. (2005) analyzed intestinal samples and found that CCL21 was expressed in the intestinal lymph nodes, lymphatic vessels, and the mesenteric lymph nodes. CCL21 was highly expressed in the HEVs, lymphatic vessels, and interstitial DC, especially in the T-cell district.

The colon submucosal layer is rich in capillary vessels. During ulcerative colitis, the expression of CCL21 is increased in the HEVs of lymph nodes, and CCR7 positive lymphocytes migrate into the lymph nodes through its concentration gradient. On the other hand, the 
number of lymphocytes and CCR7 on the lymphocytes increases during ulcerative colitis, which leads to the homing of lymphocytes, as well as to the assembly, activation, and differentiation of APCs (such as T cells and B cells) in the lymph nodes (Debes et al., 2002; Stein et al., 2003; Weninger et al., 2003; Thanarajasingam et al., 2007). CCL21 is also expressed in the rat colon, and has been suggested to be involved in the development of intestinal lymphoid tissue and regular movement. Further studies of ulcerative colitis have revealed a significant increase in the lymphocyte chemotactic effect. This effect was dose dependent and subject to saturation. Ximei Chen et al. (Chen et al., 2006) reported that ulcerative colitis significantly increased CCL21 mRNA expression in rats, which indicated that increased CCL21 expression was associated with the pathological process of ulcerative colitis. Furthermore, this study demonstrated that inhibition of CCL21 might be an effective way to reduce ulcerative colitis.

In the present study, we found that the CCL21 expression level was significantly increased in mice in the DSS group with ulcerative colitis compared to the control, which indicated a strong correlation between increases in CCL21 expression and the onset of ulcerative colitis. CCL21 expression also reflected the level of inflammation in ulcerative colitis, which could be considered as an index in evaluations of the severity of the disease. By comparing the mice in the TL and dexamethasone groups to those in the DSS group, we found that TL and dexamethasone treatments significantly decreased CCL21 expression. These treatments also reduced the clinical symptoms and local pathological damage in the mice colon, indicating that they might have therapeutic effects on ulcerative colitis through inhibition of CCL21. Therefore, CCL21 suppression could be an effective way to cure ulcerative colitis. However, compared to the control mice, mice in the TL and dexamethasone groups exhibited higher CCL21 expression, which might have been due to the co-application of DSS.

In conclusion, these results suggest that CCL21 plays an important role in ulcerative colitis. Therefore, searching for a safe and effective antagonist of CCL21 in order to decrease the inflammatory damage induced by ulcerative colitis could be a practical way to treat this disease.

\section{ACKNOWLEDGMENTS}

Research supported by the Social Application Research Plans Foundation of Nantong (\#2012074) and the Natural Science Foundation of Nantong University (\#10Z061).

\section{REFERENCES}

Ashour AE, Turnquist HR, Singh RK, Talmadge JE, et al. (2007). CCL21-induced immune cell infiltration. Int. Immunopharmacol. 7: 272-276.

Boirivant M, Fuss IJ, Ferroni L, De Pascale M, et al. (2001). Oral administration of recombinant cholera toxin subunit B inhibits IL-12-mediated murine experimental (trinitrobenzene sulfonic acid) colitis. J. Immunol. 166: 3522-3532.

Britschgi MR, Favre S and Luther SA (2010). CCL21 is sufficient to mediate DC migration, maturation and function in the absence of CCL19. Eur. J. Immunol. 40: 1266-1271.

Cheminay C, Schoen M, Hensel M, Wandersee-Steinhauser A, et al. (2002). Migration of Salmonella typhimurium harboring bone marrow - derived dendritic cells towards the chemokines CCL19 and CCL21. Microb. Pathog. 32: 207-218.

Chen XM, Ge BJ and Yang CQ (2006). Expression and effect of secondary lymphoid tissue chemokine in experimental ulcerative colitis in rats. Chin. J. Digestion 26: 614-616.

Debes GF, Hopken UE and Hamann A (2002). In vivo differentiated cytokine-producing CD4(+) T cells express functional CCR7. J. Immunol. 168: 5441-5447.

Fedele G, Bianco M, Debrie AS, Locht C, et al. (2011). Attenuated Bordetella pertussis vaccine candidate BPZE1 promotes 
human dendritic cell CCL21-induced migration and drives a Th1/Th17 response. J. Immunol. 186: 5388-5396.

Gollmer K, Asperti-Boursin F, Tanaka Y, Okkenhaug K, et al. (2009). CCL21 mediates CD4+ T-cell costimulation via a DOCK2/Rac-dependent pathway. Blood 114: 580-588.

Gunn MD, Kyuwa S, Tam C, Kakiuchi T, et al. (1999). Mice lacking expression of secondary lymphoid organ chemokine have defects in lymphocyte homing and dendritic cell localization. J. Exp. Med. 189: 451-460.

Haessler U, Pisano M, Wu M and Swartz MA (2011). Dendritic cell chemotaxis in 3D under defined chemokine gradients reveals differential response to ligands CCL21 and CCL19. Proc. Natl. Acad. Sci. U. S. A. 108: 5614-5619.

Kawashima D, Oshitani N, Jinno Y, Watanabe K, et al. (2005). Augmented expression of secondary lymphoid tissue chemokine and EBI1 ligand chemokine in Crohn's disease. J. Clin. Pathol. 58: 1057-1063.

Legler DF, Krause P, Scandella E, Singer E, et al. (2006). Prostaglandin E2 is generally required for human dendritic cell migration and exerts its effect via EP2 and EP4 receptors. J. Immunol. 176: 966-973.

Liu C, Ueno T, Kuse S, Saito F, et al. (2005). The role of CCL21 in recruitment of T-precursor cells to fetal thymi. Blood 105: 31-39.

Murano M, Maemura K, Hirata I, Toshina K, et al. (2000). Therapeutic effect of intracolonically administered nuclear factor kappa B (p65) antisense oligonucleotide on mouse dextran sulphate sodium (DSS)-induced colitis. Clin. Exp. Immunol. 120: 51-58.

Murphy PM (2010). Double duty for CCL21 in dendritic cell trafficking. Immunity 32: 590-592.

Ouyang Q, Tandon R, Goh KL, Ooi CJ, et al. (2005). The emergence of inflammatory bowel disease in the Asian Pacific region. Curr. Opin. Gastroenterol. 21: 408-413.

Riedl K, Baratelli F, Batra RK, Yang SC, et al. (2003). Overexpression of CCL-21/secondary lymphoid tissue chemokine in human dendritic cells augments chemotactic activities for lymphocytes and antigen presenting cells. Mol. Cancer 2: 35 .

Stein JV, Soriano SF, M'rini C, Nombela-Arrieta C, et al. (2003). CCR7-mediated physiological lymphocyte homing involves activation of a tyrosine kinase pathway. Blood 101: 38-44.

Stevceva L, Pavli P, Husband AJ and Doe WF (2001). The inflammatory infiltrate in the acute stage of the dextran sulphate sodium induced colitis: B cell response differs depending on the percentage of DSS used to induce it. BMC Clin. Pathol. 1: 3 .

Tal O, Lim HY, Gurevich I, Milo I, et al. (2011). DC mobilization from the skin requires docking to immobilized CCL21 on lymphatic endothelium and intralymphatic crawling. J. Exp. Med. 208: 2141-2153.

te Velde AA, de Kort F, Sterrenburg E, Pronk I, et al. (2007). Comparative analysis of colonic gene expression of three experimental colitis models mimicking inflammatory bowel disease. Inflamm. Bowel Dis. 13: 325-330.

Thanarajasingam U, Sanz L, Diaz R, Qiao J, et al. (2007). Delivery of CCL21 to metastatic disease improves the efficacy of adoptive T-cell therapy. Cancer Res. 67: 300-308.

Veerman KM, Williams MJ, Uchimura K, Singer MS, et al. (2007). Interaction of the selectin ligand PSGL-1 with chemokines CCL21 and CCL19 facilitates efficient homing of T cells to secondary lymphoid organs. Nat. Immunol. 8: 532-539.

Watanabe S, Yamakawa M, Hiroaki T, Kawata S, et al. (2007). Correlation of dendritic cell infiltration with active crypt inflammation in ulcerative colitis. Clin. Immunol. 122: 288-297.

Weninger W, Carlsen HS, Goodarzi M, Moazed F, et al. (2003). Naive T cell recruitment to nonlymphoid tissues: a role for endothelium-expressed CC chemokine ligand 21 in autoimmune disease and lymphoid neogenesis. J. Immunol. 170: 4638-4648.

Wiede F, Vana K, Sedger LM, Lechner A, et al. (2007). TNF-dependent overexpression of CCL21 is an underlying cause of progressive lymphoaccumulation in generalized lymphoproliferative disorder. Eur. J. Immunol. 37: 351-357.

Zimmerman NP, Vongsa RA, Wendt MK and Dwinell MB (2008). Chemokines and chemokine receptors in mucosal homeostasis at the intestinal epithelial barrier in inflammatory bowel disease. Inflamm. Bowel Dis. 14: 1000-1011. 